\title{
P38 MAPK signaling pathway mediates COM crystal-induced crystal adhesion change in rat renal tubular epithelial cells
}

\author{
Shiyong $\mathrm{Qi}^{1} \cdot \mathrm{Qi} \mathrm{Wang}^{1} \cdot \mathrm{Bin}_{\mathrm{Xie}}{ }^{2} \cdot$ Yue Chen $^{1} \cdot$ Zhihong Zhang $^{1} \cdot$ Yong X X $^{1}$ (i)
}

Received: 26 November 2018 / Accepted: 3 June 2019 / Published online: 10 June 2019

(c) The Author(s) 2019

\begin{abstract}
The objective of the study is to clarify the mechanism of p38 mitogen-activated protein kinase (p38 MAPK) signaling pathway in the change of crystal adhesion in rat renal tubular epithelial cells (NRK-52E) induced by calcium oxalate monohydrate (COM) crystals. NRK-52E cells were divided into COM crystal-treated group and control group according to whether the cell culture medium contains different concentrations of COM crystals. The concentrations of lactate dehydrogenase in the both group medium were determined after being cultured for $24 \mathrm{~h}$. Protein and RNA were extracted from both cell groups after being cultured at different time points. SB239063, an inhibitor of the activation of p38 MAPK, was pretreated for $2 \mathrm{~h}$ before incubation with COM crystals. Western blotting and RT-qPCR were performed to confirm the expression levels of relative genes. All the experimental results were summarized and analyzed by SPSS 20.0 statistical analysis software. COM crystals $\left(146 \mu \mathrm{g} / \mathrm{cm}^{2}\right)$ could induce the expression levels of NLRP3, caspase-1 and interleukin-1 $\beta$ (IL-1 $\beta$ ) significantly increased in NRK-52E cells. Compared with the control group cells, the transcription and translation levels of p38 MAPK-related molecule (such as p-p38) and adhesion molecules (such as osteopontin, hyaluronic acid and CD44) were significantly increased in COM crystal-treated cells and can be inhibited by SB239063 and NLRP3 gene silencing. This study demonstrated that the p38 MAPK signaling pathway mediated the COM crystal-induced crystal adhesion change in NRK-52E cells and required the involvement of NLRP3 inflammasome.
\end{abstract}

Keywords P38 MAPK $\cdot$ COM crystals $\cdot$ NLRP3 inflammasome $\cdot$ Calcium oxalate stone $\cdot$ NRK-52E

$\begin{array}{ll}\text { Abbreviations } \\ \text { p38 MAPK } & \text { p38 mitogen-activated protein kinase } \\ \text { COM } & \text { Calcium oxalate monohydrate } \\ \text { LDH } & \begin{array}{l}\text { Lactate dehydrogenase } \\ \text { NLRP3 }\end{array} \\ \begin{array}{l}\text { Nucleotide binding oligomerization domain- } \\ \text { like receptor protein } 3\end{array} \\ \text { HLP } & \begin{array}{l}\text { Hydroxy-L-proline } \\ \text { HA }\end{array} \\ \text { Hyaluronic acid }\end{array}$

Shiyong Qi and Qi Wang contributed equally to this work.

Electronic supplementary material The online version of this article (https://doi.org/10.1007/s00240-019-01143-z) contains supplementary material, which is available to authorized users.

Yong $\mathrm{Xu}$

drxuyong1955@126.com

1 Department of Urology, Tianjin Institute of Urology, The Second Hospital of Tianjin Medical University, 23 Pingjiang Road, Hexi District, Tianjin 300211, China

2 Department of Surgery, Linyi People's Hospital, Linyi, Shandong, China

$\begin{array}{ll}\text { HAS } & \text { Hyaluronan synthases } \\ \text { OPN } & \text { Osteopontin } \\ \text { FBS } & \text { Fetal bovine serum } \\ \text { P/S } & \text { Penicillin/streptomycin } \\ \text { siRNA } & \text { Small interfering RNA } \\ \text { SEM } & \text { Scanning electron microscopy } \\ \text { RT-qPCR } & \text { Real-time quantitative polymerase chain } \\ & \text { reaction } \\ \text { NC } & \text { Negative control } \\ \text { ERK1/2 } & \text { Extracellular-signal regulated kinase 1/2 } \\ \text { JNK } & \text { c-Jun N-terminal kinase } \\ \text { p-p38 } & \text { Phospho-p38 } \\ \text { PDTC } & \text { Pyrrolidine dithiocarbamate } \\ \text { ER } & \text { Mineralocorticoid receptor } \\ \text { MDCK } & \text { Madin-Darby canine kidney } \\ \text { IL-1 } \beta & \text { Interleukin-1 } \beta\end{array}$




\section{Introduction}

Renal stones are one of the most common diseases in urology; about $5 \%$ of American males will be involved in their lifetime, and the percentage is still increasing [1]. Calcium oxalate is one of the most common components of kidney stones and involved in the formation of $80 \%$ renal stones [2]. Studies had demonstrated that the damage of renal tubular epithelial and the change of crystal adhesion in renal tubular epithelial cells were the key chains in the formation of calcium oxalate stones [3, 4]. However, the underlying mechanisms participating in the change of crystal adhesion in renal tubular epithelial cells were still unclear.

The nucleotide-binding oligomerization domain-like receptor protein 3 (NLRP3) inflammasome is a wellknown cytoplasmic protein complex that associated with various metabolic diseases [5-7]. Knauf et al. found that, compared with wild-type mice, the incidence of oxalate nephropathy and renal failure was significantly reduced in NLRP $3^{-1-}$ mice that fed with the diet high in soluble oxalate [8]. Mulay et al. demonstrated that renal inflammation induced by calcium oxalate crystals was mediated by NLRP3 inflammasome [9]. Joshi et al. found that the deposition of calcium oxalate crystals and the expression of NLRP3 in renal tissues were elevated in kidneys of rats fed with hydroxy-L-proline (HLP) [10]. However, the role of the NLRP3 inflammasome in the adhesion of COM crystals in the renal tubular epithelium was still unclear and need further researches to verify.

The adhesion of COM crystals to renal tubular epithelial cells is the basis for the onset of calcium oxalate stone disease. Former studies had illustrated that hyaluronic acid (HA) was a high molecular mass polysaccharide and upregulated in kidney tissue during inflammation [11, 12]. Hyaluronan synthases (HAS) was a key enzyme for HA synthesis and had three isoenzymes, termed as HAS1, HAS2 and HAS3, respectively [13]. Osteopontin (OPN) was a kind of glycoprotein and was significantly up-regulated during inflammation [13]. Studies had demonstrated that CD44 was a cell surface receptor for HA and OPN and also up-regulated during renal inflammation [13, 14]. Marino Asselman et al. demonstrated that the retention of calcium oxalate crystals required renal tubular epithelial damage and the expression of HA, OPN and CD44 in the rat kidneys [15].

P38 MAPK, one of the isozymes of MAPK, participated in the onset of atherosclerosis by directly affecting the expression of collagen [16]. The study of Paleerath et al. illustrated that the disruption of tight junction in epithelial cells was mediated by p38 MAPK signaling pathway and the expression level of related proteins of p38 MAPK signaling pathway were up-regulated during calcium oxalate stone formation [17]. On these bases, we could hypothesize that the COM crystal-induced overexpression of adhesion molecules are mediated by $\mathrm{p} 38$ MAPK signaling pathway and NLRP3 inflammasome plays an important role in it.

\section{Materials and methods}

\section{Preparation of COM crystals}

The preparation of COM crystals was referenced from the study of Thongboonkerd et al. [18]. Calcium chloride dihydrate $(10 \mathrm{mM})$ was mixed with sodium oxalate $(10 \mathrm{mM})$, and a mixture of a final concentration of $5 \mathrm{mM}$ and $0.5 \mathrm{mM}$ was prepared in Tris buffer containing $90 \mathrm{mM} \mathrm{NaCl}$. The mixture was incubated overnight at $25{ }^{\circ} \mathrm{C}$ and COM crystals were harvested by centrifugation at $3000 \mathrm{rpm}$ for $5 \mathrm{~min}$. The supernatant was discarded and the crystals were re-suspended in methanol. After further centrifugation at $3000 \mathrm{rpm}$ for $5 \mathrm{~min}$, methanol was discarded and the crystals were dried overnight at $37^{\circ} \mathrm{C}$. Then the COM crystals were decontaminated by UV light for $30 \mathrm{~min}$. Finally, they were added to complete Eagle's minimum essential medium (MEM) to reach a final concentration of $1000 \mu \mathrm{g} / \mathrm{ml}$.

\section{Cell culture}

The rat kidney proximal tubular epithelial (NRK-52E) cells were purchased from SIBS (Shanghai Institutes for Biological Sciences). NRK-52E cells were cultured in high-glucose DMEM supplemented with $10 \%$ fetal bovine serum (FBS) and $1 \%$ penicillin/streptomycin $(\mathrm{P} / \mathrm{S})$ under $5 \% \mathrm{CO}_{2} / 95 \%$ atmosphere at $37{ }^{\circ} \mathrm{C}$ incubator, and serial sub-cultivation in T-25 flasks. DMEM, FBS, penicillin and streptomycin were bought from Gibco (USA), NRK-52E cells were cultured in 6- and 24-well plates. COM crystals were added at final different concentrations of $0,36.5,73.0,109.5,146$, and $182.5 \mu \mathrm{g} / \mathrm{cm}^{2}$, respectively. NRK-52E cells grew to $70-80 \%$ confluence in complete medium and the different concentrations of COM crystals were added, after incubated for 0,12 and $24 \mathrm{~h}$, the cells and medium were separately collected for further analysis. SB239063 (purchased from Sigma), an inhibitor of the activation of p38 MAPK, was prepared in PBS. SB239063 was added at final different concentrations of $20 \mathrm{mM}$ and incubated with NRK-52E cells for $2 \mathrm{~h}$ and then the cells were collected for further analysis.

\section{LDH release}

LDH released into cell culture medium was induced by cell membrane injury. NRK-52E cells were seeded in six-well 
plate as mentioned above and COM crystals were added at final different concentrations of $0,36.5,73.0,109.5,146.0$, $182.5 \mu \mathrm{g} / \mathrm{cm}^{2}$. After incubating with cells for $24 \mathrm{~h}, \mathrm{LDH}$ was measured by LDH-kit (Nanjing Jiancheng Bioengineering Institute) as per the constructor's protocol. The final result was measured by Microplate Reader at $450 \mathrm{~nm}$.

\section{Immunofluorescence staining}

NRK-52E cells were seeded in 24-well plates via the method of coverslip culture, while NRK-52E cells grew to 30-50\% confluence in complete medium, COM crystals were added at final concentration of $146.0 \mu \mathrm{g} / \mathrm{cm}^{2}$ and incubated for $24 \mathrm{~h}$. After incubation overnight with primary antibodies anti-NLRP3 (1:50, Abcam, Cambridge, UK) and anti-caspase-1 (1:50, Abcam) and then incubating with secondary antibodies, images were obtained with the fluorescence microscope (Olympus, Tokyo, Japan).

\section{Small interfering RNA (siRNA) knockdown experiments}

Double-stranded siRNA targeting NLRP3 gene and a negative control (NC) siRNA were purchased from GenePharma (Shanghai, China). NRK-52E cells were transfected using siRNA mixed with Lipofectamine 2000 (Invitrogen, USA) following the manufacturer's protocol. The NLRP3-siRNA sequence is $5^{\prime}$-GGAGAGACCUUUAUGAGAATT-3' and 5'-UUCUYCAUAAGGUCUCUCCTG-3' as a reverse sequence. Negative control sequence is $5^{\prime}$-UUCUCCGAA CGUGUCACGUTT- 3 ' and the reverse sequence is $5^{\prime}$-ACG UGACACGUUCGGAGAATT-3'.

\section{Western blotting analysis}

Protein extractives were lysed in mammalian cell lysis buffer. Protein concentration was determined referring to a bovine serum albumin standard. Proteins were separated on 10-12\% SDS-PAGE gels. Protein expression levels were determined using primary antibodies anti-NLRP3 (1:500, ab214185), anti-caspase-1 (1:500, ab108362), anti-IL-1 $\beta$ (1:500, ab2105), anti-HAS1 (1:500, ab198846), anti-p-p38 (1:500, ab45381) and anti-p38 (1:500, ab31828), which were purchased from Abcam (Cambridge, UK), and primary antibodies anti-CD44 (1:1000, 15675-1-AP), anti-OPN (1:1000, 22952-1-AP) were purchased from Proteintech (USA). Antirabbit and anti-mouse IgG secondary antibodies were purchased from Santa Cruz (USA). GAPDH (Santa Cruz, USA) was used as loading control.

\section{Scanning electron microscopy (SEM)}

Experimental NER-52E cells were seeded in 24-well plates via the method of coverslip culture. The cells were treated with or without COM crystals for about $24 \mathrm{~h}$ and COM crystal exposure cells were also divided into two groups according to whether pretreatment with SB239063 at $20 \mathrm{mM}$ for $2 \mathrm{~h}$, and then all cells were fixed with glutaraldehyde at $4{ }^{\circ} \mathrm{C}$ refrigerator through the night. After that, the cells were viewed and imaged using a Hitachi S-3400 N SEM.

\section{Real-time quantitative polymerase chain reaction (RT-qPCR) analysis}

The total RNA from NRK-52E cells were extracted using the Trizol Reagent (Invitrogen, USA), and cDNA was then synthesized using a reverse transcription (RT) system kit (Thermo Fisher Scientific, USA) according to the manufacturer's instructions. RT-qPCR was performed using the SYBR Premix Ex Taq II (Takara Biotechnology, China) following the manufacturer's protocols. The sequences of related primers used in this study are shown in Table 1 . The amplification and analysis were performed on a Real-Time PCR system (ABI prism 7500, Applied Biosystems, USA). All of the data were analyzed with $-\Delta \Delta C_{t}$ method and normalized using GAPDH cDNA as an internal control.

Table 1 Sequences of related primers for research

\begin{tabular}{lll}
\hline & Forward & Reverse \\
\hline NLRP3 & 5'-CGTGAGTCCCATTAAGATGGAGT-3' & 5'-CCCGACAGTGGATATAGAACAGA-3' \\
P38 & 5'-TTTCCGCAAGGTTCGATTTTCA-3' & 5'-GGCATCTGCGCTCTACCATC-3' \\
HAS1 & 5'-CACTGTGTATCCTGCATCAG-3' & 5'-CTTGGTAGCATAACCCATGC-3' \\
HAS2 & 5'-CTCAGTGTTATACATGTCGAGTTTACTTC-3' \\
HAS3 & 5'-AGTGCAGCTTCGGGGATGA-3' & 5'-ACTGATACTGGAATGAGTCCTATGAA-3' \\
QPN & 5'-AGCACAGCATCGTCGGGAC-3' & 5'-TGATGGTAGCAATGGCAAAGAT-3' \\
CD44 & 5'-CTGTCTGTGCTGTCGGTGAT-3' & 5'-TCCTTGGTCGGCGTTTGGCTG-3' \\
\hline
\end{tabular}




\section{Results}

\section{COM crystals could induce the formation of NLRP3 inflammasome}

The intracellular lactate dehydrogenase (LDH) was released into the cell culture medium when cells were dead and we indirectly measured the effect of different concentrations of COM crystals on cell death by detecting the concentration of $\mathrm{LDH}$ in the cell culture medium. The concentration of LDH in culture medium was detected after NRK-52E cells were incubated with different concentrations $(0,36.5,73.0,109.5$, $146.0,182.5 \mu \mathrm{g} / \mathrm{cm}^{2}$ ) of COM crystals for $24 \mathrm{~h}$. The results revealed that, compared with low concentrations $(36.5,73.0$, $109.5 \mu \mathrm{g} / \mathrm{cm}^{2}$ ) of COM crystals, the cell death significantly increased when incubated with higher concentrations (146.0, $182.5 \mu \mathrm{g} / \mathrm{cm}^{2}$ ) of COM crystals (Fig. 1a). By measuring the expression level of NLRP3 in COM-treated NRK-52E cells, we found that COM crystals $\left(146.0 \mu \mathrm{g} / \mathrm{cm}^{2}\right)$ could induce the transcription and translation level of NLRP3 significantly increased (Fig. 1b, c, e). IL-1 $\beta$ has been illustrated to be activated by the activation of NLRP3 inflammasome [19] and we also found the expression level of it also significantly increased in COM crystal-treated cells (Fig. 1b). Procaspase-1, a component of NLRP3 inflammasome, can be cleaved into its active form caspase- 1 by the formation of NLRP3 inflammasome [20]. Our study also demonstrated that COM crystals $\left(146.0 \mu \mathrm{g} / \mathrm{cm}^{2}\right)$ could induce the production of caspase-1 (Fig. 1e). From the above findings, we concluded that $\mathrm{COM}$ crystals could induce the formation of NLRP3 inflammasome in NRK-52E cells.
A

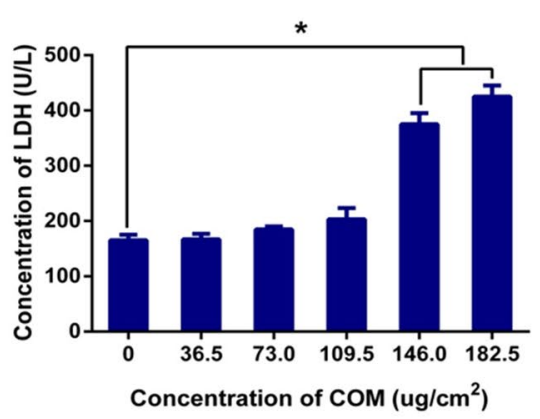

B
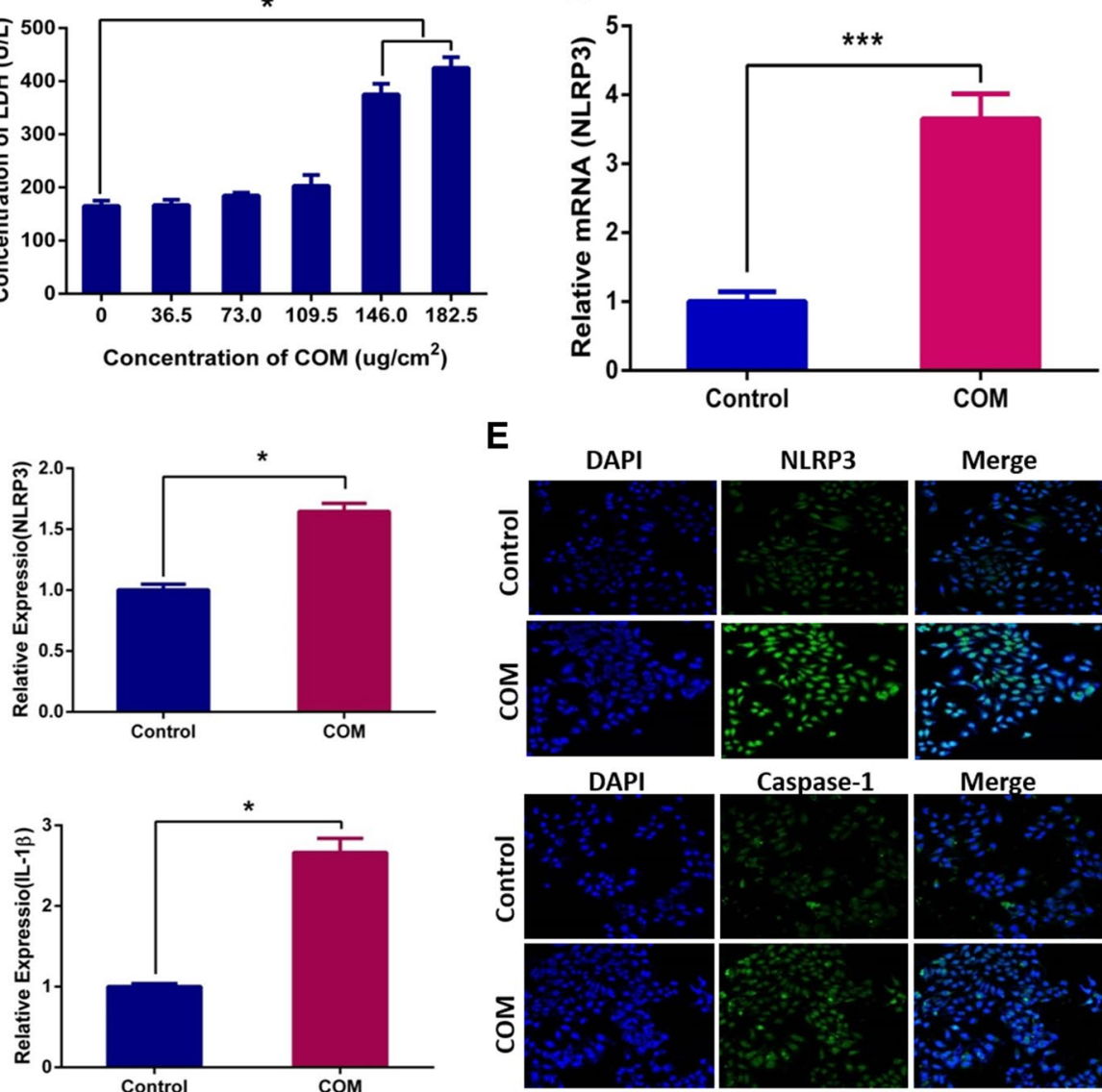

E DAPI

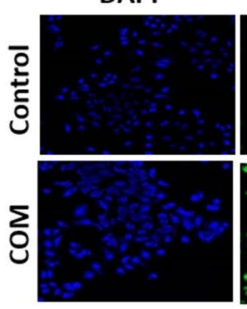

DAPI

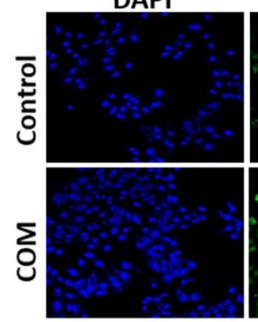

NLRP3

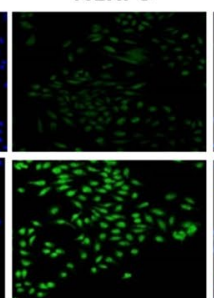

Caspase-1

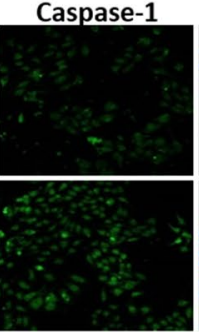

C

NLRP3

IL-1及

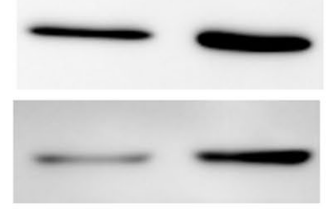

GAPDH
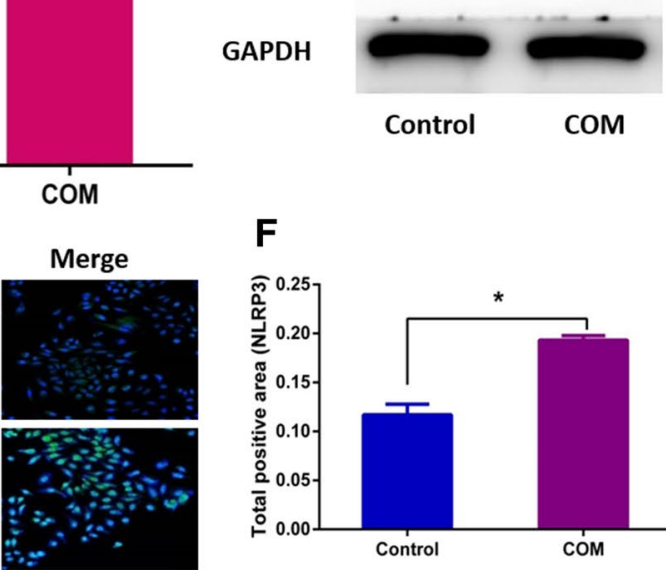

Merge

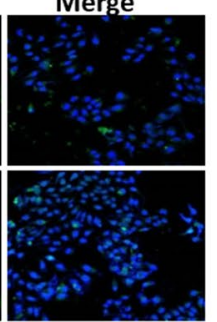

$\mathbf{F}$
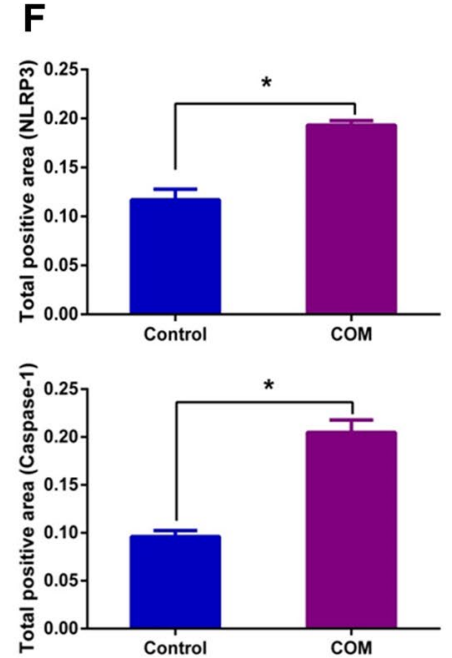

Fig. 1 COM crystal could induce the formation of NLRP3 inflammasome. a Detection of the concentrations of LDH in NRK-52E cells culture medium performed after NRK-52E cells were incubated with differential concentration of COM crystals for $24 \mathrm{~h}$. $* P<0.05$; b RTqPCR was used to detect the expression level of NLRP3 mRNA in NRK-52E cells exposed with COM $\left(146.0 \mu \mathrm{g} / \mathrm{cm}^{2}\right)$, whereas GAPDH served as the loading control. $* * * P<0.005$; c Western blotting was used to measure the expression levels of NLRP3 and IL-1 $\beta$ in NRK-52E cells exposed to COM $\left(146.0 \mu \mathrm{g} / \mathrm{cm}^{2}\right)$, GAPDH served as the loading control; $\mathbf{d}$ band intensities of NLRP3 and IL-1 $\beta$, respectively, were measured by a densitometer and normalized with that of GAPDH, $* P<0.05$; e immunofluorescence staining analysis of the expression levels of NLRP3 (green) and caspase-1 (green) in NRK52E cells exposed with COM $\left(146.0 \mu \mathrm{g} / \mathrm{cm}^{2}\right)$, the nucleuses were stained with DAPI (blue) and the magnification of all images is 200; $\mathbf{f}$ total positive area of NLRP 3 and caspase- 1 were measured by Image $\mathrm{J}$ software, ${ }^{*} P<0.05$. $N=3$ independent experiments for each bar 


\section{SB239063 can inhibit the COM crystal-induced activation of p38 MAPK signaling pathway in NRK-52E cells}

Phospho-p38 (p-p38), the active form of p38, was performed to examine the effects of COM crystals on p38 MAPK signaling pathway in NRK-52E cells. The expression level of total p-38 was directly detected by $\mathrm{p}-38$. The data revealed that COM crystal exposure of NRK-52E cells had induced the expression level of p-p38 significantly increased. SB239063, a specific inhibitor of the activation of p38 MAPK signaling pathway, was non-toxic to NRK-52E cells (Fig. 2a), was treatment with cells for $2 \mathrm{~h}$ at $20 \mathrm{mM}$ before the incubation of COM crystals. According to the results, we found that the expression level of p-p38 was significantly increased when incubated with COM crystals for 12 or $24 \mathrm{~h}$, meanwhile the expression level of p38 has not changed (Fig. 2b). Thus, we concluded that COM crystals could activate p38 MAPK signaling pathway by inducing the phosphorylation of $\mathrm{p} 38$. At the same time, our study also found that SB239063 can effectively inhibit the COM crystal-induced up-regulation of the expression level of p-p38 (Fig. 2b). OPN, a kind of adhesion molecule, was up-regulated in NRK-52E cells when incubated with COM crystals for 12 or $24 \mathrm{~h}$, and which also could be suppressed by SB239063 (Fig. 2b). Thus, we concluded that the COM crystal-induced change of crystal adhesion in NRK-52E cells was mediated by $\mathrm{p} 38$ MAPK signaling pathway and can be prevented by SB239063.

\section{NLRP3 gene silencing can prevent the COM crystal-induced overproduction of NLRP3, p-p38 and OPN in NRK-52E cells}

The above experiments have demonstrated that the p38 MAPK signaling pathway plays an important role in the COM crystal-induced change of crystals adhesion in NRK52 cells; however, the relationship between NLRP3 inflammasome and p38 MAPK signaling pathway was still unclear. First of all, we found that the cell viability of NRK-52E
A

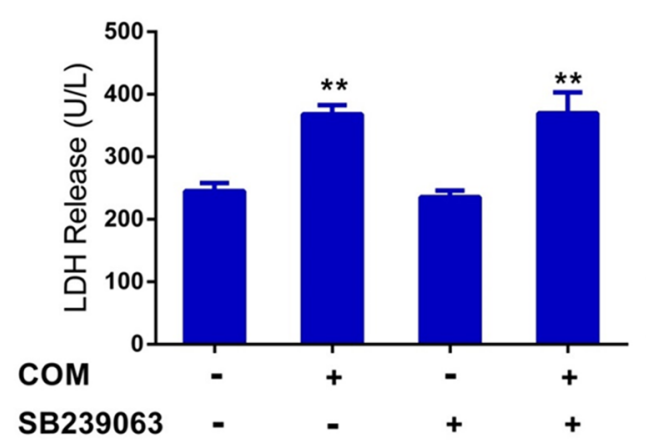

B

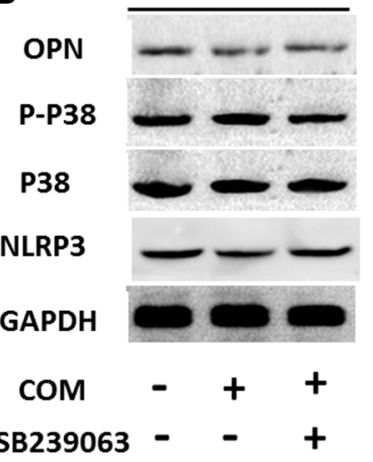

$12 \mathrm{~h}$

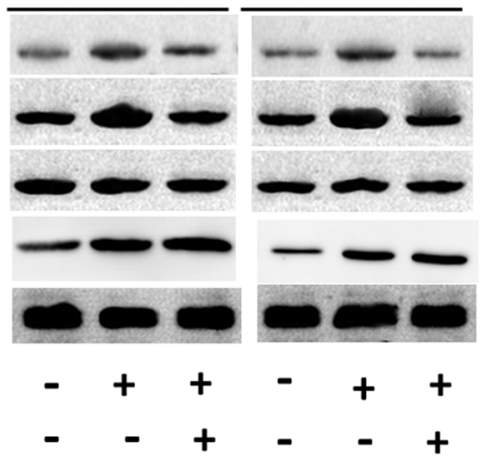

C Control $\square$ COM $\square$ SB239063.

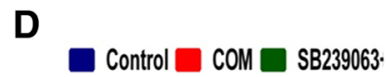

E
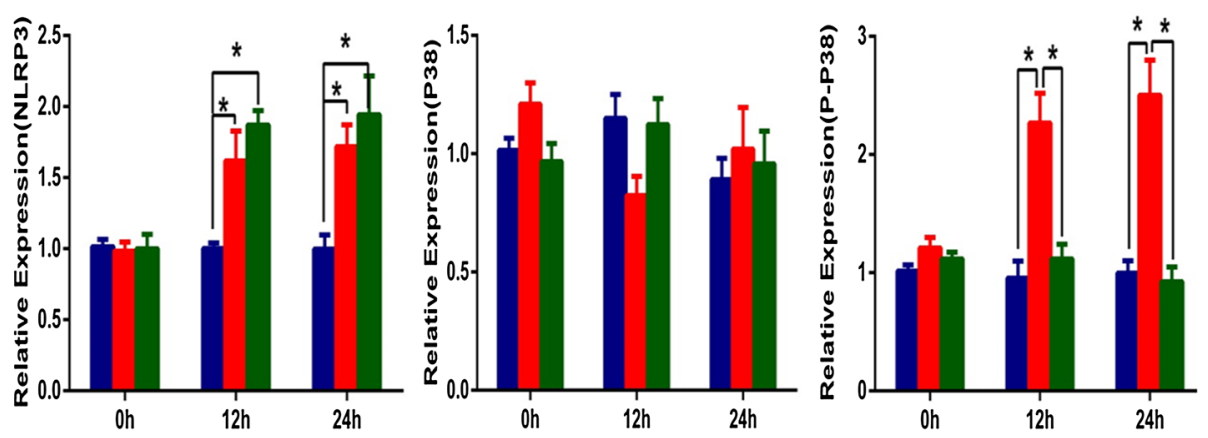

$\mathbf{F}$

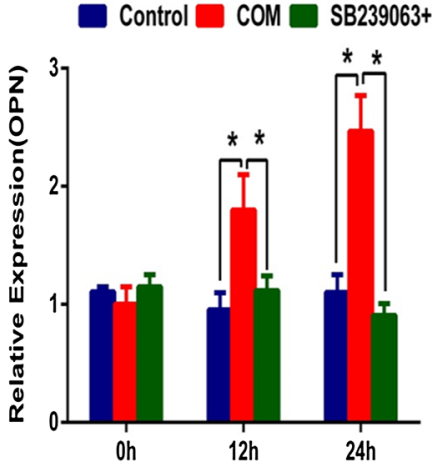

Fig. 2 Expression levels of related proteins in SB239063-treatedNRK-52E cells. a The cell toxicity of SB239063 was detected by LDH-kit; $* * P<0.01$ VS. control group; b proteins derived from whole cell lysates of controlled and COM-treated $\left(146.0 \mu \mathrm{g} / \mathrm{cm}^{2}\right)$ cells at different time points $(0,12,24 \mathrm{~h})$, without or with 2-h pretreatment by $20 \mathrm{mM}$ SB239063 (an inhibitor of p38MAPK activa-

tion), were equally loaded into each of SDS-PAGE lane. The proteins were subjected to Western blotting analysis including anti-NLRP3, anti-p38, anti-p-p38 and anti-OPN. GAPDH served as the loading control. c-f Band intensities of NLRP3, p38, p-p38 and OPN, respectively, were measured by a densitometer and normalized with GAPDH. $N=3$ independent experiments for each bar; $* P<0.05$ 


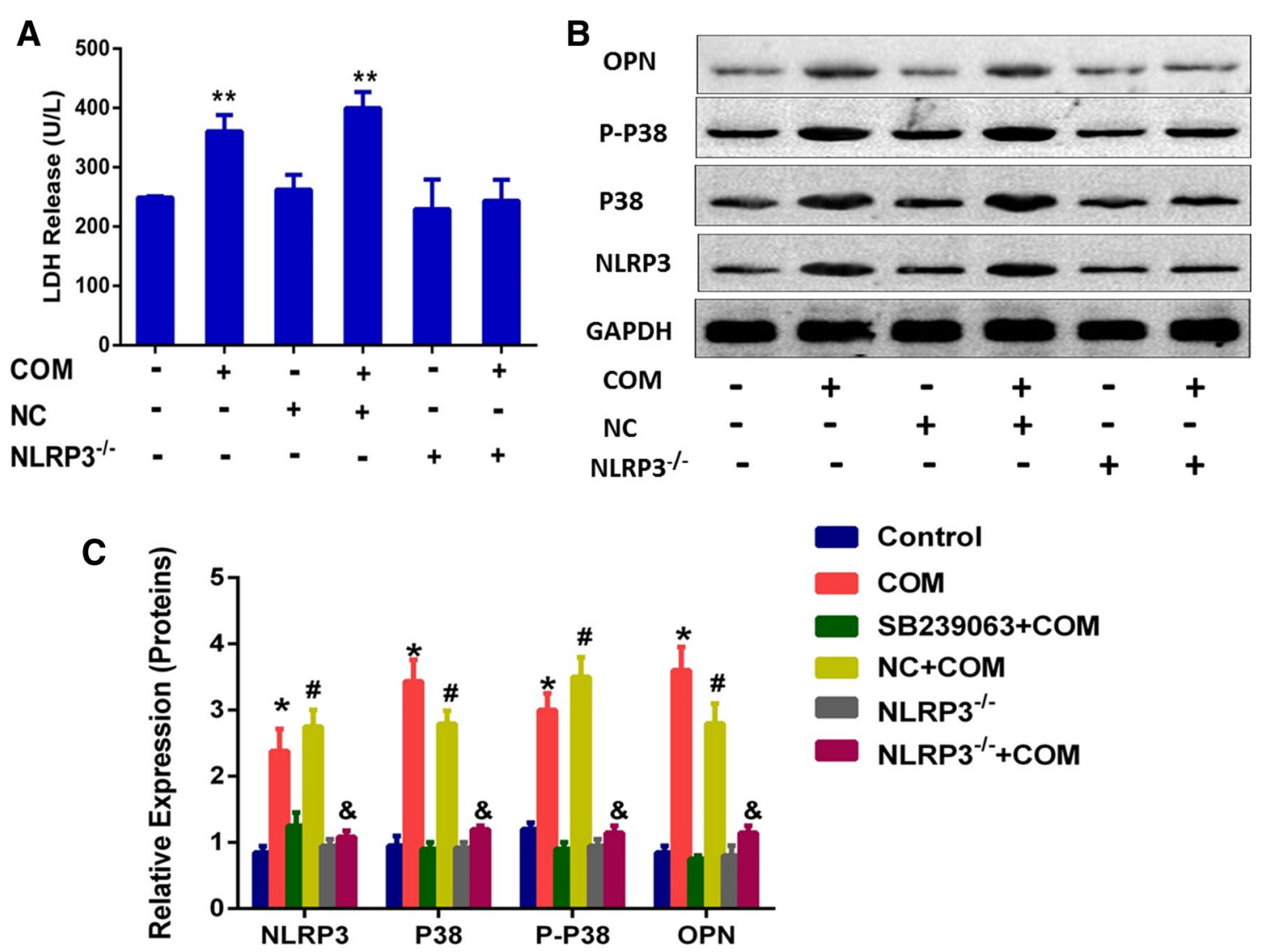

Fig. 3 Expression levels of related proteins in NLRP3 gene-silenced NRK-52E cells. a The cell toxicity of NLRP3-siRNA was detected by LDH-kit; ${ }^{* *} P<0.05$ vs. control group; $\mathbf{b}$ proteins derived from whole cell lysates of controlled and COM-treated $\left(146.0 \mu \mathrm{g} / \mathrm{cm}^{2}\right)$ cells without or with transfection of NLRP3-SiRNA or NC, were equally loaded into each of SDS-PAGE lane. The resolved proteins were then subjected to Western blot analysis for NLRP3, p38, p-p38 and OPN, whereas GAPDH served as the loading control. c Band intensities of NLRP3, total p38, phospho-p38 and OPN, respectively, were measured by a densitometer and normalized with GAPDH. $N=3$ independent experiments for each bar; $* P<0.05$ vs. control group; ${ }^{\#} P<0.05$ vs. NC group; $* * P<0.05$ vs. COM group or $\mathrm{COM}+\mathrm{NC}$ group

crystal treatment could increase the expression levels of other adhesion molecules, such as CD44 and HA. We indirectly reflect the expression level of HA by detecting the expression levels of HAS1, HAS2 and HAS3. Western blotting and RT-qPCR analysis were used to detect the expression levels of adhesion molecules in COM crystaltreated NRK-52E cells. The data revealed that the COM crystal exposure of NRK-52E cells could induce the transcription levels of HAS1, HAS2, HAS3, CD44 and OPN significantly increased, whereas SB239063 or NLRP3 gene silencing could prevent the COM crystal-induced overproduction of adhesion molecules on the surface of NRK-52E cells (Fig. 4a). Meanwhile, we also detected the translation levels of OPN, HAS1 and CD44 and found that the COM crystal-induced overexpression of OPN, HAS1 and CD44 in NRK-52E cells could be prevented by NLRP3 gene silencing and SB239063 (Fig. 4b). Thus, we can make a conclusion that NLRP3 inflammasome and p38 MAPK signaling pathway involved in the COM 

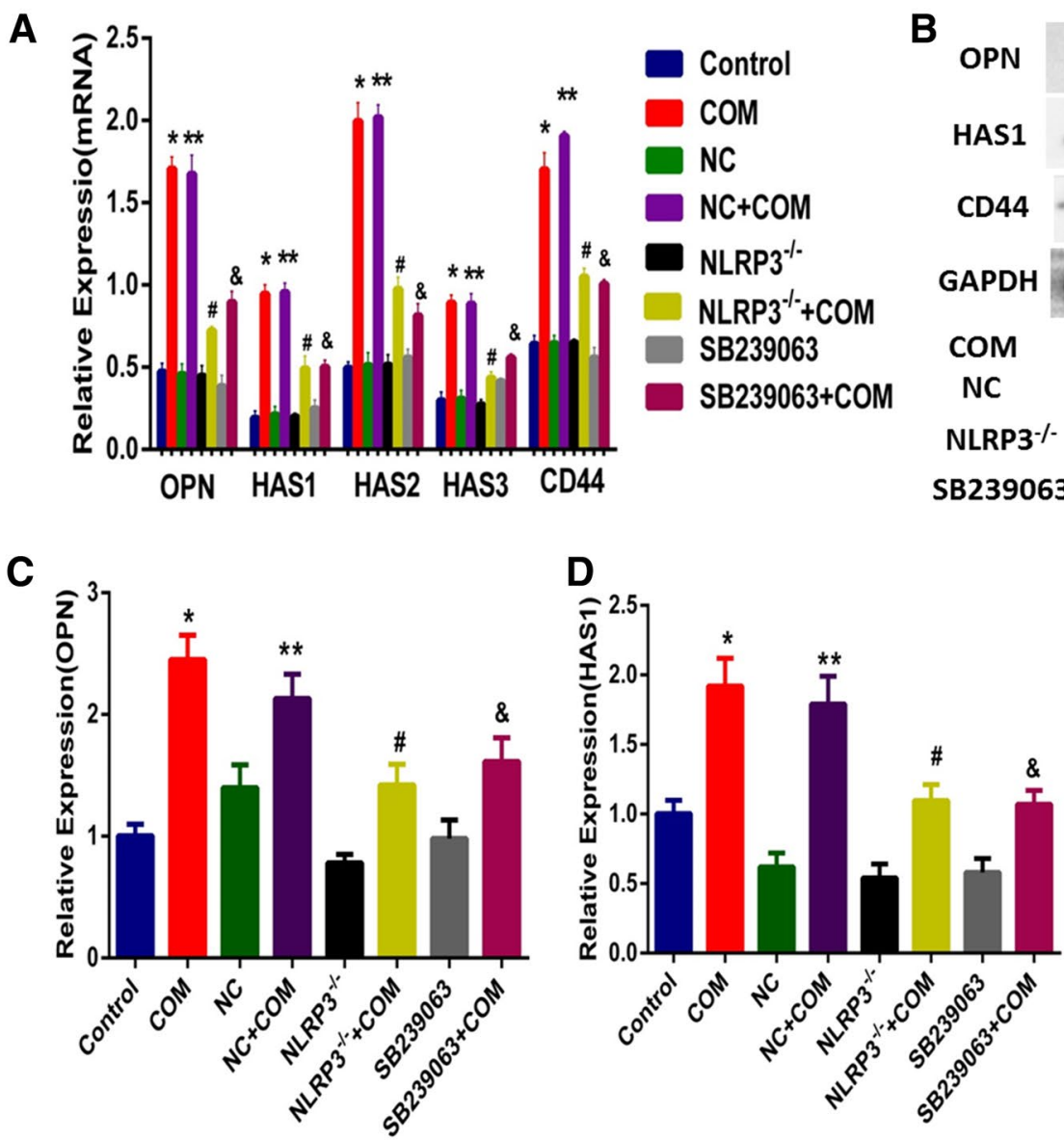

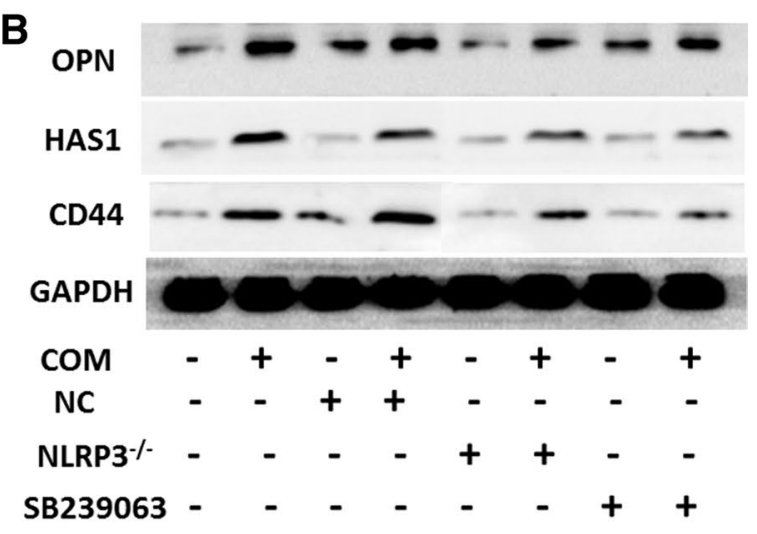

E

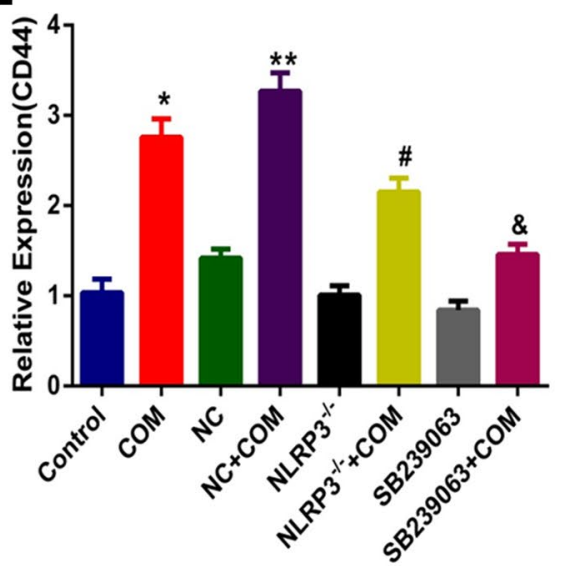

Fig. 4 Detecting the expression levels of adhesion molecules. a Total RNA from whole cell lysates with different treatment, cDNA were synthesized according to the procedure of HiFiScript cDNA Synthesis Kit. RT-qPCR was used to detect the expression levels of HAS1, HAS2, HAS3, CD44 and OPN mRNA in NRK-52E cells exposed with COM $\left(146.0 \mu \mathrm{g} / \mathrm{cm}^{2}\right)$, whereas GAPDH served as the loading control. $N=3$ independent experiments for each bar; $* P<0.05$ vs. control; ${ }^{* *} P<0.05$ vs. NC group; ${ }^{\#} P<0.05$ vs. COM group or $\mathrm{COM}+\mathrm{NC}$ group; ${ }^{\&} P<0.05$ vs. $\mathrm{COM}$ group or $\mathrm{COM}+\mathrm{NC}$; b Western blot analysis for CD44, HAS1 and OPN in NRK-52E cells exposed with COM $\left(146.0 \mu \mathrm{g} / \mathrm{cm}^{2}\right)$ and GAPDH served as

crystal-induced overproduction of adhesion molecules in NRK-52E cells.

\section{Visualization of crystal adhesion change in NRK-52E cells}

The mechanism of COM crystal-induced crystal adhesion change in NRK-52E cells has been preliminary demonstrated; however, the microscopic performance of the crystal adhesion change in NRK-52E cells was still unclear. Control group cells and COM-treated NRK-52E cells, with or without the pretreatment of SB239063, were observed by SEM. Compared with the control group cells, we found that the COM crystal-treated cells had obvious morphological the loading control. c Band intensities of CD44, HAS1 and OPN, respectively, were measured by a densitometer and normalized with GAPDH, $N=3$ independent experiments for each bar; $* P<0.05$ vs. control; ${ }^{*} P<<0.05$ vs. NC group; ${ }^{\#} P<0.05$ vs. COM group or $\mathrm{COM}+\mathrm{NC}$ group; ${ }^{\circledR} P<0.05$ vs. $\mathrm{COM}$ group or $\mathrm{COM}+\mathrm{NC} ; \mathbf{c}-\mathbf{e}$ band intensities of OPN, HAS1, and CD44, respectively, were measured by a densitometer and normalized with GAPDH. $N=3$ independent experiments for each bar; $* P<0.05$ vs. control; $* * P<0.05$ vs. NC group; ${ }^{\#} P<0.05$ vs. $\mathrm{COM}$ group or $\mathrm{COM}+\mathrm{NC}$ group; ${ }^{\&} P<0.05$ vs. COM group or $\mathrm{COM}+\mathrm{NC}$

changes which were characterized by cell edema and the COM crystal adhesion to the cell membrane significantly increased. Meanwhile, the COM crystal-treated cells pretreated with SB239063 did not show the above changes (Supplementary Fig. 1).

\section{Discussion}

P38 MAPK, a 38KD protein composed of 360 amino acids, combined with extracellular-signal regulated kinase $1 / 2$ (ERK1/2) and c-Jun N-terminal kinase (JNK) to form the three major branches of MAPK signaling system [21]. P38 MAPK signaling pathway, composed of MAPK kinase 
kinase, MAPK kinase and p38 MAPK, was an important member of the MAPK family. Under the stimulation of various factors, such as hyperglycemia, pro-inflammatory factors, oxidative stress, the phosphorylation of MAPK kinase as followed by the phosphorylation of MAPK kinase and finally activated the p38 MAPK by inducing the phosphorylation of p38 MAPK residues [22]. The activated p38 MAPK signaling pathway participate in the processes of cell growth, differentiation, apoptosis, environmental stress response and inflammatory response [23].

Several studies had demonstrated that oxalate selectively activated the p38 MAPK signaling pathway and the activation of $\mathrm{p} 38$ MAPK signaling pathway was required for the re-initiation of oxalate-induced DNA synthesis in renal epithelial cells $[24,25]$. The study of Han et al. found that oxalate could inhibit the proliferation of renal proximal tubule cells via the p38 MAPK/JNK signaling pathway and which can be prevented by the inhibitor of p38 MAPK signaling pathway [26]. Recent study also illustrated that the p38 MAPK pathway plays an important role in regulating the nephrotoxicity of oxalate in human renal epithelial cells [27]. Meanwhile, the study of Ilbey et al. demonstrated that pyrrolidine dithiocarbamate (PDTC) could prevent the deposition of crystals in renal tubules by reducing the expression levels of oxidative stress, iNOS, NF-kappa B and p38 MAPK [28]. Based on the above results, we could suspect that the p38 MAPK signaling pathway plays an important role in COM crystal-induced change of crystals adhesion in NRK-52E cells.

NLRP3 inflammasome, a kind of protein complex existing in the cytoplasm, includes NLRP3 receptor, apoptosisassociated speck-like protein containing CARD (ASC) and procaspase-1 [8]. The formation of NLRP3 inflammasome will consequently induce procaspase- 1 cleaved into its active form caspase-1 [20, 29]. IL-1 $\beta$, a kind of interleukin, was involved in the onset and progression of inflammation [30]. Studies have demonstrated that the activation of NLRP3 inflammasome will induce pro-IL- $1 \beta$ cleaved into its mature form IL-1 $\beta[9,19]$. Previous study has indicated that calcium oxalate crystals could induce innate immunity mediated by NLRP3/ASC/caspase-1 axis and IL-1 $\beta$ in intrarenal mononuclear phagocytes [9]. Our study has demonstrated that COM crystals could induce the overexpression of NLRP3, caspase- 1 and IL- $1 \beta$, which indirectly reflects the formation of NLRP3 inflammasome.

It is well known that the enhancement of adhesion between COM crystals and renal tubular epithelial cells was one of the key points in the onset and progression of calcium oxalate stones. OPN, CD44 and HA were the most studied adhesion molecules. The study of Asselman et al. found the damage of tubular epithelial and the over-expression of HA, OPN and CD44 in rat kidney with oxalate crystal deposition [15]. Then the study of Tsuji et al. suggested that the inhibition of OPN expression in the renal of hyperoxaluric rats also inhibited the deposition of renal crystals [31]. These results indicated that the enhanced adhesion of calcium oxalate crystals to renal tubular epithelial cells was achieved by the crystal-induced over-expression of adhesion molecules. At the same time, study also demonstrated that the aldosterone-induced expression of OPN in vascular smooth muscle cells was mediated by mineralocorticoid receptor (ER) and the involved signaling cascades including ERK and p38 MAPK [32]. The study of Zuo et al. illustrated that the atorvastatin-induced expression of OPN could be prevented by the inhibition of the p38 MAPK signaling pathway [33]. Recent study suggested that ROS-Akt-p38 MAPK signaling pathway was activated in COM crystal-induced disruption of tight junction in Madin-Darby canine kidney (MDCK) cells [34]. These data confirmed the hypothesis that the COM crystal-induced activation of $\mathrm{p} 38$ MAPK signaling pathway was associated with the change of crystal adhesion in NRK$52 \mathrm{E}$ cells.

Our study demonstrated that COM crystals could induce the over-expression of OPN in NRK-52E cells and this result was consistent with the former studies. In our study, we also found that COM crystals could induce the expression level of p-p38 significantly which directly demonstrated the activation of p38 MAPK signaling pathway. However, the overexpression of OPN and p-p38 can be prevented by the activation inhibitor of $\mathrm{p} 38$ MAPK signaling pathway. These results were consistent with other studies and proved that the up-regulation of adhesion molecules in NRK-52E cells induced by COM crystals was mediated by p38 MAPK signaling pathway. Other studies also had proved that NLRP3 inflammasome plays an important role in the pathogenesis of oxalate-induced nephropathy [8-10]. Our study also illustrated that NLRP3 gene silencing could suppress the upregulation of OPN and p-p38, which directly proved that the COM crystal-induced crystal adhesion change was mediated by $\mathrm{p} 38$ MAPK signaling pathway and p38 MAPK signaling pathway was the downstream of NLRP3 inflammasome. Our study first reported the intuitive morphological changes of crystal adhesion in NRK-52E cells by scanning electron microscopy, and found that the changes can be abrogated by the activation inhibitor of p38 MAPK signaling pathway.

Based on our research and previous reports, the underlying mechanism of crystal adhesion change in NRK-52E cells induced by COM crystals can be explained as follows. First, the COM crystal incubation with the cell culture medium could induce the up-regulation of NLRP3 inflammasome in NRK-52E cells and then NLRP3 inflammasome activated the p38 MAPK signaling pathway by inducing the phosphorylation of p38. Finally, high expression levels of adhesion molecules were mediated by $\mathrm{p} 38$ MAPK signaling pathway thereby changing the adhesion of COM crystals in NRK52E cells. The enhancement of COM crystal adhesion in 
NRK-52E cells could lead to the increase of COM crystals adhering to the renal tubular epithelium. With large COM crystal particles being formed, the calcium oxalate stones will be formed eventually.

Our study found that the reductions of NLRP3 inflammasome and p38 MAPK signaling pathway productions in NRK-52E cells were associated with the reduction of COM crystals adhering to NRK-52E cells. Our study also illustrated that the enhancement of crystal adhesion to NRK-52E cells induced by COM crystals was mediated by NLRP3 inflammasome and p38 MAPK signaling pathway and the underlying mechanisms in the onset and progression of calcium oxalate stones were further demonstrated. However, our study is not devoid of limitations. First, the results of in vitro experiments lack the validation of animal experiments. Second, although it has been illustrated that the COM crystal-induced change of crystal adhesion in NRK-52E cells requires the involvement of NLRP3 inflammasome and p38 MAPK signaling pathway, it is still unclear whether other signaling molecules or signaling pathways were involved in this process. Finally, various factors were involved in the formation of calcium oxalate stones in human kidneys and our study only simulates the effect of COM crystal exposure on NRK-52E cells. Therefore, additional studies should be performed to verify the specific underlying mechanisms of the crystal adhesion change in NRK-52E cells.

In summary, our study points out the important roles of p38 MAPK signaling pathway and NLRP3 inflammasome in COM crystal-induced change of crystal adhesion in NRK52E cells. Our research has preliminary proved the pathogenesis of calcium oxalate stone disease, which provides a direction for the following researches in specific underlying mechanisms and the new ideas for the prevention and treatment of calcium oxalate stone disease.

Author contributions SQ conceived the project. SQ, QW, BX and YC conceived and designed the experiments. QW and $\mathrm{BX}$ performed the experiments. SQ, ZZ and YX analyzed the data. SQ, QW, and BX wrote the paper.

Funding This work was supported by the Science and Technology Project of Tianjin (17ZXMFSY00060), the Key Laboratory Fund Project of the Second Hospital of Tianjin Medical University (2017ZDSYS14) and the Education Commission Research Project of Tianjin (2017KJ208). The funder had no role in study design, data collection and analysis, decision to publish, or preparation of the manuscript.

\section{Compliance with ethical standards}

Conflict of interest The authors declare that they have no conflict of interest.

Ethical approval This article does not contain any studies with human participants or animals performed by any of the authors.
Open Access This article is distributed under the terms of the Creative Commons Attribution 4.0 International License (http://creativeco mmons.org/licenses/by/4.0/), which permits unrestricted use, distribution, and reproduction in any medium, provided you give appropriate credit to the original author(s) and the source, provide a link to the Creative Commons license, and indicate if changes were made.

\section{References:s}

1. Stamatelou KK, Francis ME, Jones CA et al (2003) Time trends in reported prevalence of kidney stones in the United States: 1976-1994. Kidney Int 63(5):1817-1823

2. Coe FL, Parks JH, Asplin JR (1992) The pathogenesis and treatment of kidney stones. N Engl J Med 327(16):1141-1152

3. Jonassen JA, Cooney R, Kennington L et al (1999) Oxalateinduced changes in the viability and growth of human renal epithelial cells. J Am Soc Nephrol 10(Suppl 14):S446-S451

4. Khan SR (1995) Calcium oxalate crystal interaction with renal tubular epithelium, mechanism of crystal adhesion and its impact on stone development. Urol Res 23(2):71-79

5. Wree A, Eguchi A, McGeough MD et al (2014) NLRP3 inflammasome activation results in hepatocyte pyroptosis, liver inflammation, and fibrosis in mice. Hepatology 59(3):898-910

6. Peng K, Liu L, Wei D et al (2015) P2X7R is involved in the progression of atherosclerosis by promoting NLRP3 inflammasome activation. Int J Mol Med 35(5):1179-1188

7. Luo B, Li B, Wang W et al (2014) NLRP3 gene silencing ameliorates diabetic cardiomyopathy in a type 2 diabetes rat model. PLoS One 9(8): 104771

8. Knauf F, Asplin JR, Granja I et al (2013) NALP3-mediated inflammation is a principal cause of progressive renal failure in oxalate nephropathy. Kidney Int 84(5):895-901

9. Mulay SR, Kulkarni OP, Rupanagudi KV et al (2013) Calcium oxalate crystals induce renal inflammation by NLRP3-mediated IL-1beta secretion. J Clin Investig 123(1):236-246

10. Joshi S, Wang W, Peck AB et al (2015) Activation of the NLRP3 inflammasome in association with calcium oxalate crystal induced reactive oxygen species in kidneys. J Urol 193(5):1684-1691

11. Knepper MA, Saidel GM, Hascall VC et al (2003) Concentration of solutes in the renal inner medulla: interstitial hyaluronan as a mechano-osmotic transducer. Am J Physiol Ren Physiol 284(3):F433-F446

12. Johnsson C, Tufveson G, Wahlberg J et al (1996) Experimentally-induced warm renal ischemia induces cortical accumulation of hyaluronan in the kidney. Kidney Int 50(4):1224-1229

13. Auvinen P, Rilla K, Tumelius R et al (2014) Hyaluronan synthases (HAS1-3) in stromal and malignant cells correlate with breast cancer grade and predict patient survival. Breast Cancer Res Treat 143(2):277-286

14. Aruffo A, Stamenkovic I, Melnick M et al (1990) CD44 is the principal cell surface receptor for hyaluronate. Cell 61(7):1303-1313

15. Asselman M, Verhulst A, De Broe ME et al (2003) Calcium oxalate crystal adherence to hyaluronan-, osteopontin-, and CD44-expressing injured/regenerating tubular epithelial cells in rat kidneys. J Am Soc Nephrol 14(12):3155-3166

16. Zhao W, Ma G, Chen X (2014) Lipopolysaccharide induced LOX-1 expression via TLR4/MyD88/ROS activated p38MAPKNF-kappaB pathway. Vascul Pharmacol 63(3):162-172

17. Peerapen P, Thongboonkerd V (2013) p38 MAPK mediates calcium oxalate crystal-induced tight junction disruption in distal renal tubular epithelial cells. Sci Rep 3:1041 
18. Singhto N, Sintiprungrat K, Sinchaikul S et al (2010) Proteome changes in human monocytes upon interaction with calcium oxalate monohydrate crystals. J Proteome Res 9(8):3980-3988

19. Haldar S, Dru C, Choudhury D et al (2015) Inflammation and pyroptosis mediate muscle expansion in an interleukin-1beta (IL-1beta)-dependent manner. J Biol Chem 290(10):6574-6583

20. He WT, Wan H, Hu L et al (2015) Gasdermin D is an executor of pyroptosis and required for interleukin-1beta secretion. Cell Res 25(12):1285-1298

21. Brewster JL, de Valoir T, Dwyer ND et al (1993) An osmosensing signal transduction pathway in yeast. Science 259(5102):1760-1763

22. Ono K, Han J (2000) The p38 signal transduction pathway: activation and function. Cell Signal 12(1):1-13

23. Schett G, Zwerina J, Firestein G (2008) The p38 mitogen-activated protein kinase (MAPK) pathway in rheumatoid arthritis. Ann Rheum Dis 67(7):909-916

24. Chaturvedi LS, Koul S, Sekhon A et al (2002) Oxalate selectively activates p38 mitogen-activated protein kinase and c-Jun $\mathrm{N}$-terminal kinase signal transduction pathways in renal epithelial cells. J Biol Chem 277(15):13321-13330

25. Koul HK, Menon M, Chaturvedi LS et al (2002) COM crystals activate the p38 mitogen-activated protein kinase signal transduction pathway in renal epithelial cells. J Biol Chem 277(39):36845-36852

26. Han HJ, Lim MJ, Lee YJ (2004) Oxalate inhibits renal proximal tubule cell proliferation via oxidative stress, p38 MAPK/ JNK, and cPLA2 signaling pathways. Am J Physiol Cell Physiol 287(4):C1058-C1066

27. Koul S, Khandrika L, Pshak TJ et al (2014) Oxalate upregulates expression of IL-2Rbeta and activates IL-2R signaling in HK-2 cells, a line of human renal epithelial cells. Am J Physiol Renal Physiol 306(9):F1039-F1046
28. Ilbey YO, Ozbek E, Simsek A et al (2010) Pyrrolidine dithiocarbamate treatment prevents ethylene glycol-induced urolithiasis through inhibition of NF-kappaB and p38-MAPK signaling pathways in rat kidney. Arch Ital Urol Androl 82(2):87-94

29. Abdul-Muneer PM, Alikunju S, Mishra V et al (2017) Activation of NLRP3 inflammasome by cholesterol crystals in alcohol consumption induces atherosclerotic lesions. Brain Behav Immun 62:291-305

30. Darisipudi MN, Thomasova D, Mulay SR et al (2012) Uromodulin triggers IL-1beta-dependent innate immunity via the NLRP3 inflammasome. J Am Soc Nephrol 23(11):1783-1789

31. Tsuji H, Shimizu N, Nozawa M et al (2014) Osteopontin knockdown in the kidneys of hyperoxaluric rats leads to reduction in renal calcium oxalate crystal deposition. Urolithiasis 42(3):195-202

32. Fu GX, Xu CC, Zhong Y et al (2012) Aldosterone-induced osteopontin expression in vascular smooth muscle cells involves MR, ERK, and p38 MAPK. Endocrine 42(3):676-683

33. Zuo L, Du Y, Lu M et al (2014) Atorvastatin inhibits hyperglycemia-induced expression of osteopontin in the diabetic rat kidney via the p38 MAPK pathway. Mol Biol Rep 41(4):2551-2558

34. Yu L, Gan X, Liu X et al (2017) Calcium oxalate crystals induces tight junction disruption in distal renal tubular epithelial cells by activating ROS/Akt/p38 MAPK signaling pathway. Ren Fail 39(1):440-451

Publisher's Note Springer Nature remains neutral with regard to jurisdictional claims in published maps and institutional affiliations. 